\title{
Review of: "Olfactory detection and discrimination in domestic dogs (Canis lupus familiaris)"
}

Tina Bloom

Potential competing interests: The author(s) declared that no potential competing interests exist.

Review of Detection and Discrimination in Domestic Dogs (Canis lupus familiaris)

Which was written by Ferrando and Dahl (2022)

Review by

Tina Bloom ${ }^{1,2,3}$

\footnotetext{
${ }^{1}$ New York State Licensed Psychologist, NYS Forensic Outreach \& Crisis Team, West Seneca, NY, USA ${ }^{2}$ Department of Psychology \& Counseling, Floraglades, Foundation, Ft. Myers, FL, USA

3 Bahnhof Dobermans \& Lionbacker Rhodesian Ridgebacks, Venus, PA, USA
}

\section{Introduction}

Some argue that the notion of dogs scenting better than humans is a $19^{\text {th }}$ Century myth (Barwich, 2016). In fact, it has been claimed that for certain scent detection purposes, human olfaction ability may be equal to or better than that of dogs, if only humans ambulated on all four limbs, which would place human noses closer to the ground (Porter et al., 2007). The idea that human scenting ability surpasses or even compares to canine scent detection capability could be disposed of rather quickly by testing whether humans could detect a bomb (Furton \& Myers, 2001) in a passerby's suitcase when crawling through an airport on all fours, or perhaps testing whether a human, after practicing enough, could detect the location of a nest of insects (Moser et al., 2020) when walking into an infested home. Such investigations would likely readily confirm Lazarowski and colleagues' (2020) well-founded warning that methodology must be closely evaluated when investigating scent detection.

In an effort to use a solid methodological foundation, Ferrando and Dahl applied classic psychophysics as a means to study scent detection ability in dogs. However, their methodology could be further refined by moving from the 1800's psychophysics approach of Weber's law (Fechner, 1860) into modern Signal Detection Theory (SDT; Swets, 1961, 1979; Swets et al., 1961). SDT more precisely dissects the nonlinear differences between physical stimuli and the perception of those stimuli by quantifying subjective organismic (internal) and environmental (external) noise in the system as well as perceiver bias, all of which affects signal detection. Additionally, collaboration with canine scent detection and discrimination trainers would facilitate fine-tuning of stimuli and stimulus presentation.

\section{Summary of Ferrando \& Dahl}


Ferrando and Dahl accurately and painstakingly described Weber's Just Noticeable Difference (JND; Fechner,1860), which is a measure of the perception of change in stimulus intensity. The JND is a measure of subjectivity and not a measure of physical intensity, which is objective. In essence, early psychophysicists found that there was not a linear relationship between objective measures of physical stimuli intensity and their subjective experiences (perception). For example, if one sees a candle in the distance, it does not take much light (perhaps one more candle) to notice this difference in stimulus intensity. However, if one sees a spotlight in the distance, the addition of a single candle's light beside it would never be noticed. Classic psychophysicists found that the larger the stimulus, the larger the difference that must be presented for an individual to be capable of noticing the change in intensity, and thus, the term JND, represented by the equation:

$$
\frac{\Delta I}{I}=k
$$

In this equation, I is a variable that represents the measure of intensity of the stimulus. It was found that $k$ tends to remain constant across intensities within a single perceptual channel (i.e., vison, audition, scent, and taste, as well as the thermoreceptors and mechanoreceptors of tactile somatosensory channels). In early psychophysics, human vison or audition were the typically investigated sensory modalities. Although constant within a given sensory channel, $k$ was found to differ across the various senses.

In addition to Weber's JND, Ferrando and Dahl briefly described Roberts's (2010) approximate number system (ANS) to address findings in nonhuman animals' ability to detect quantitative differences and Gestalt laws in psychology (Ginsburg \& Nicholls, 1988) to further address subjectivity in the canine perception of scent. Ferrando and Dahl astutely acknowledged Horowitz and collaborators' (2013) finding that, on average, dogs paid more attention to a covered plate with more food ( 5 units) than one baited with less food (1 unit). Ironically, dogs in Horowitz and colleagues' investigation did not select the largerbaited plate significantly more frequently than the less-baited plate. Ferrando and Dahl also compared several dog breeds as well as examined differences when these breeds were grouped into categories reflecting three different skull morphologies (i.e., cephalic indexes of brachycephalic, mesocephalic, and dolichocephalic).

Ferrando and Dahl had three hypotheses. First, they hypothesized it would be easier for dogs to discriminate between food and no food than it would be for them to discriminate between a larger and a smaller amount. Second, they hypothesized longer-nosed (dolichocephalic) dogs would perform better than shorter-nosed (brachycephalic) dogs, with of course, medium-nosed (mesocephalic) dogs performing in the middle. Third, they hypothesized Weber's Law would apply to canine olfactory discrimination in general and that some breeds would be more capable of discriminating between larger and smaller differences in quantity. They used a two-alternative forced choice protocol to test these hypotheses. In general, Ferrando and Dahl found that dogs did better at detection than discrimination. Breed differences were found in both tasks, with Jack Russel Terriers excelling on both tasks while all but one Bichon Bolognese (who achieved $100 \%$ correct) performed at or below chance responding on both tasks. 
While the authors acknowledged that their cephalic classification did not follow scientifically accepted classifications of breeds into cephalic categories, given their limited number of breeds, their measure of cephalic index appeared to interact with type of task (detection vs. discrimination). Although the longednosed (dolichocephalic) dogs tended to perform better at both tasks, the difference was not statistically different across cephalic index for the discrimination task. There appears to be an error in the manuscript text on lines 346 to 349; on line 348, the word "discrimination" should be the word "detection" (shown below):

This interaction manifested in an increase in performance scores with increasing cephalic index class, i.e., from brachycephalic to dolichocephalic in the discrimination [author italics; should this word be detection?] task, but remains at similar levels across cephalic index classes in the discrimination task.

\section{Suggestions for Improvement in Future Research}

\section{Signal Detection Theory (SDT)}

Detection and discrimination between quantities of olfactory stimuli are perceptual tasks, in which a perceiver (in this case a domestic canine) attempts to determine the presence of a signal or a difference between two signals. Signal Detection Theory (SDT) explains how errors occur during such perceptual processes (Swets, 1961, 1979; Swets et al., 1961). Prior to SDT, psychophysicists considered only two states of the world: the target signal was present, or it was not (Fechner, 1860/1966). However, this simplistic conceptualization was problematic in that sometimes, when an easily detectible signal was present, individuals did not perceive it; and conversely, when no signal was present, at times, people reported observing one (Green \& Swets, 1966). Thus, the need for quantification of subjective experiences that accounts for errors. Ferrando and Dahl attempted to examine canine ability to detect (or discriminate between) signals by utilizing concepts from Weber (the JND), ANS, and Gestalt Psychology. Perhaps SDT would be another productive route for them to pursue.

To explain such errors, Swets and colleagues proposed that perception is based on the detection of an informational pattern (signal) that may or may not be present in the environment. In the real world, the presence of a signal is a condition that we do not ever know for certain; therefore, a signal's presence can be represented as a probability distribution. Additionally, there is always noise, which can be environmental (e.g., in visual tasks, fog or a dirty glasses) or organismic (e.g., in visual tasks, blinking one's eyes or intruding internal stimuli). Just as the signal's possible presence is represented by a probability distribution, noise can also be represented as a probability distribution. Thus, signal and noise can be illustrated as two overlapping bell curves with the signal being on the right and the noise being on the left. In SDT, the perceptual system's sensitivity ( $d^{\prime}$, or the ability to detect signals) is influenced by both environmental and organismic factors that can either enhance or diminish the perceptual process.

Sensitivity $\left(d^{\prime}\right)$ is determined by the distance between the means of the noise distribution and the signal distribution.

The measure of bias $(\beta)$ is independent of the sensitivity of the system and defined as the probability of the 
willingness to say, "Yes, the signal is present," despite its actual presence. Bias $(\beta)$ acts as a criterion that can move to the left or right, across both overlapping distributions (of signal and noise). Values to the left of $\beta$ are deemed to be "not present," while values to the right of $\beta$ are deemed to be "present." As $\beta$ moves to the left, miss rate (one of the two possible errors) decreases, but as correct hit rate increases, so does the incorrect false alarm rate. As $\beta$ moves to the right, false alarm rate (the other possible error) decreases, but as correct rejection rates increase, so does the miss rate. Without improving sensitivity $\left(d^{\prime}\right)$ by spreading the means of the signal and noise distributions further apart (e.g., in visual perception, getting glasses or in auditory perception, getting a hearing aid), it is impossible to increase one type of correct response without also increasing one type of incorrect response, which reflects the reality of the perceptual process, and subsequent decisions regarding the presence or absence of a signal. Bias $(\beta)$ offers a way to represent the perceptual set of the perceiver.

In perception, there will always be uncertainty and its concomitant error. Bias determines the rates of the types of errors made in any given situation. Bias is directed by perceived costs and benefits. For example, if a false alarm occurs much too often when there appears to be a minimum chance of real danger, resulting in a perceived waste of valuable time for naught (e.g., the smoke detector goes off every time I cook causing me to get a broom and wave it by the detector for several minutes), then I set my bias accordingly (e.g., I think, "Well, there never is a REAL fire"). Sometimes, the result is that I make a very poor decision, (e.g., I take the battery out of the smoke detector). There will be no more false alarms, but neither will there be warning of a real fire!

When the cost of a miss feels high, for example, when a missed signal leads to much discomfort such as getting soaked by an unexpected storm on the way to work, I stand much more chance of minimizing the discomfort of a false alarm error. Thus, I think, "I see a cloud; it might rain." Consequently, I carry an umbrella on mostly sunny days. I may be labeled as obsessive, but if carrying an umbrella does not inconvenience me, I choose to allow a high false alarm rate, because the cost is low, while the cost of a miss is being soaked all day long at work.

Viewing dogs' scenting ability from the perspective of SDT would acknowledge that their perceptual system quickly makes decisions about targets even when targets are not present. Additionally, when targets are present, they can be missed due to noise in the system. Furthermore, the criteria for choosing present or not present (or selecting a larger over a smaller quantity) would involve the bias of the dog. The use of trials with no stimuli present would help determine dogs' tendencies to indicate presence of a signal when there is no signal. For example, in Ferrando and Dahl's study, the Jack Russel Terriers outperformed all other breeds. Jack Russel Terriers are known for their high energy levels. Would they display more false alarms than the other breeds if they were placed in a situation where they were asked to detect a stimulus, but there was no stimulus? Perhaps Jack Russel terriers appear to be doing better as a result of their activity level (a type of bias), which would lead to a higher probability of indicating a target? Conceivably, their superior performance may not due be to their superior detection and discrimination abilities? Utilizing a SDT protocol, future studies could answer this question, and perhaps this possibility 
should be mentioned in Ferrando and Dahl's discussion section.

As well, observing dogs' tendencies when using visual stimuli (which are much more comfortable for human witnesses) would contribute to identifying dogs' behavioral tendencies prior to their assessment of scenting abilities. Petrazzini and colleagues' (2020) visual protocol could be used to establish dogs' propensities to choose more or less baited plates prior to testing them on scent discrimination tasks. Finally, to assess sensitivity, training dogs in detection and discrimination tasks so that changes in their behaviors under various environmental conditions that may lead to environmental noise (e.g., temperature, temperature changes, barometric pressure, humidity, sunlight exposure, etc.), all of which affect scent dispersion and concentration (Gerritsen \& Haak, 2016; Schoon \& Haak, 2002), could be examined.

A major concern of canine detection trainers is that shorter-nosed dogs are known to have respiratory difficulties and decreased ability to cool themselves during exertion or bouts of hot weather (Gerritsen \& Haak, 2016). Scent work exacerbates dogs' danger of overheating because when they open their mouths to cool themselves, scenting ability concomitantly wanes. Due to the need for a closed mouth when efficiently scenting, even under normal weather conditions, scent work is extremely strenuous for dogs causing them to quickly reach their physiological limits. Brachycephalic dogs are at a disadvantage in scenting tasks due to their need for "more rest stops on long tracks" (Gerritsen \& Haak, 2016, pp. 205 206). Working dog trainers and handlers avoid shorter-nosed breeds for these very practical reasons. Thus, the cephalic index may have nothing to do with scenting ability, but instead physical limitations (a kind of organismic noise) dependent upon ambient temperature and humidity (a kind of environmental noise). Future studies could test this hypothesis, and perhaps the importance of such study of environmental factors interacting with canine morphology could be mentioned in Ferrando and Dahl's discussion section.

\section{SDT Implications for Canine Scent Detection}

Without understanding specific dogs' behavioral tendencies and physical limitations, it would be difficult to tease apart scenting ability from non-scenting-related lack of physical ability or motivation to perform the task. For example, the abysmal performance of most of the Bichon Bolognese in Ferrando and Dahl's study leads one to believe that this breed simply cannot perform this task. However, the only perfect performance in both detection and discrimination tasks was achieved by one of the Bichon Bolognese. Clearly, Bichon Bolognese's anatomy and physiology could perform the task because one of them completed both the detection and the discrimination tasks perfectly, which leads to several questions. Was this one Bichon Bolognese endowed with scenting superpowers, or possibly, some unknown physical or motivational characteristic that allowed this particular Bichon Bolognese to overcome the possible discomfort associated with a brachycephalic anatomy (and possibly reducing the organismic noise of discomfort in this specific dog). Another possibility could be that the other poor performing Bichon Bolognese were affected by unnoticed environmental factors, such as barometric pressure, temperature, and humidity that conspired against all of them, except the one overperforming Bichon Bolognese that 
happened to run through the experiment at an optimal time. In future studies, these questions could be answered, and perhaps should be asked in Ferrando and Dahl's discussion section.

Alternatively, was the lack of performance in the majority of Bichon Bolognese due to a canine bias of some sort? Perhaps they had no appetite (and thus, no motivation to search for food). Did they have a behavioral tendency not to cooperate? As a person who has trained hundreds of tracking and scent detection dogs, I have found that about three in ten very good scent detection dogs will decide to check where they know the scent is not located, before they then decide to go where the scent is located, and then perfectly indicate its presence. Perhaps these Bichon Bolognese merely wanted to check the "other" place first, and they were discontinued before they were finished? Such idiosyncratic behaviors in scent detection dogs, especially breeds not known for their working abilities or trainability, must be considered in order to reduce confounds. Because many of these questions cannot be answered by Ferrando and Dahl's work, the discussion section could note such possibilities for future research.

Fortunately, in the tightly controlled environment of a laboratory experiment, researchers can objectively and systematically tease apart confounding variables, such as environmental factors (noise) from a specific dog's selection patterns (organismic noise and bias). Future research could establish specific canine participants patterns of responding to visual stimuli and then use these patterns as a separate factor to consider when investigating scent detection. Moreover, perhaps dogs of numerous breeds could be trained to detect various scents, and then their typical performance styles during training could be used as a factor in later tests of detection and discrimination. Such controls would begin to illuminate the many factors that affect canine search strategies.

\section{Olfaction is Unique}

Humans are visually oriented animals who typically pay little attention to scent (Gerritsen \& Haak, 2016). Schoon and Haak (2002, p. 52 - 63), authored a book entitled K-9 Suspect Discrimination: Training and Practicing Scent Identification Lineups, a task in which dogs match items found at crime scenes to humans in a lineup, clearly demonstrating the acuity of canine scent detection. Schoon, a Dutch-trained zoologist, who teamed up with Haak, a Dutch canine officer, found dogs' scent matching of items to suspects to be more reliable than fingerprints in suspect discrimination. These researchers noted that light, a visual stimulus, can be quantified by the objective parameters of wavelength and lux. Sound, an auditory stimulus, can be measured by the objective parameters of frequency and decibels. Odor, however, cannot be measured so easily.

Additionally, odor is affected by processes that do not noticeably affect stimuli to which humans typically attend. Thus, humans can fail to account for factors such as time elapsed since placement of the stimulus, barometric pressure, temperature, temperature change, humidity, location of the body where the item had been touched, and even sunlight exposure. The scent discrimination ability of dogs was found to be extremely reliable when dogs are properly trained, as was evidenced by Schoon and Haak (2002, p. 69) finding that dogs were able to distinguish between identical twins with diet differences $83.5 \%$ of the time. 
When identical twins ate the same diet, dogs' correct choices fell to $49 \%$ of the time. Such astute dissection of the parameters of stimulus odors illustrates the importance of researchers' collaboration with working dog trainers and handlers in order to adequately and accurately operationalize variables and their levels in scent detection and discrimination investigations.

\section{Conclusions}

It is well known among those who train and handle scent detection dogs, that not only does handler knowledge of scent location (DeChant et al., 2020) affect scent detection performance but also the handler's general understanding of scent behavior under various environmental conditions is critical in real world scent detection (Gerritsen \& Haak, 2016; Schoon \& Haak, 2002). Moreover, specific anatomical characteristics in canines that affect ability to perform scent work (e.g., length of mouth and tongue necessary for cooling during the strenuous work of scenting), and behavioral idiosyncrasies (biases such as motivation to work and energy level) within canines when working are critical in the assessment of canine ability to perform scent detection and discrimination (Gerritsen \& Haak, 2016). Although the Ferrando and Dahl investigation utilizes classic psychophysics (Weber's JND), approximate number system (ANS), and Gestalt Psychology to examine subjective aspects of canine olfactory perception, which is an excellent beginning, it is suggested that in future study, they use more sophisticated methods, such as Signal Detection Theory (SDT) to account for environmental and organismic noise as well as bias in the dogs being examined. Additionally, collaboration with effective dog trainers and handlers who specialize in scent detection and scent discrimination is highly recommended because of their astute, skill-based knowledge of scent characteristics and scent movement as well as their familiarity with scent detection and discrimination dogs. Such recommendations could be made in Ferrando and Dahl's discussion section.

\section{References}

Barwich, A. (2016). Making sense of smell: Ann-Sophie Barwich on why philosophers should pay attention to olfactory perception. The Philosopher's Magazine, 73(2), 41-47. https://doi.org/10.5840/tpm20167370 DeChant, M. T., Ford, C., \& Hall, N. J. (2020). Effect of handler knowledge of detection task on canine search behavior and performance. Frontiers in Veterinary Science. doi.org/10.3389/fvets.2020.00250 Fechner, G. T. (1860). Elemente der psychophysik, vol. 2. Breitkopf u. Hartel. Ferrando, E., \& Dahl, C. D. (2022). Olfactory detection and discrimination in domestic dogs (Canis lupus familiaris). BioRxiv, (Article being reviewed).

Furton, K. G., \& Myers, L. J. (2001). The scientific foundation and efficacy of the use of canines as chemical detectors for explosives. Talanta, 54(3), 487-500. doi.org/10.1016/S0039-9140(00)00546-4

Gerritsen, R., \& Haak, R. (2016). K9 investigation errors. Edmonton, AB: Brush Education, Inc. Ginsberg, N., \& Nicholls, A. (1988). Perceived numerosity as a function of item size. Perceptual and Motor Skills, 67(2), 656-658.

Green, D. M., \& Swets, J. A. (1966). Signal detection theory and psychophysics. New York: Wiley. 
Horowitz, A., Hecht, J., \& Dedrick, A. (2013). Smelling more or less: Investigating the olfactory experience of the dog. Learning and Motivation, 44(4), 2007-2017.

Lazarowski, L., Krichbaum, S., De Greef, L. E., Simon, M. S., Singletary, M., Angle, C., Waggoner, L. P. (2020). Methodological considerations in canine olfactory research. Frontiers in Veterinary Science. doi.org/10.3389/fvets.2020.00408.

Moser, A. Y., Brown, W. Y., Bizo, L. A., Andrew, N. R., \& Taylor, M. K. (2020). Biosecurity dogs detect live insects after training with odor-proxy training aids: Scent extract and dead specimens. Chemical Senses, 45(3), 179-186. doi: 10.1093/chemse/bjaa001

Porter, J., Craven, B., Rehan, M. K., Shao-Ju, C., Kang, I., Judkewitz, B., Volpe, J., et al. (2007). Mechanisms of scent-tracking in humans. Nature Neuroscience, 10, 27-29. https://www.nature.com/articles/nn1819 Petrazzini, M. E. M., Mantese, F., \& Prato-Previde, E. Food quantity discrimination in puppies (Canis lupus familiaris). Animal Cognition, 23(1095). doi:10.1007/s10071-020-01378-z

Roberts, W.A. (2010). Distance and magnitude effects in sequential number discrimination by pigeons. Journal of Experimental Psychology: Animal Behavior Processes, 36(2). 206.

Schoon, A., \& Haak, R. (2002). K9 suspect discrimination: Training and practicing scent detection line-ups. Calgary, AB: Detselig Enterprises, LTD.

Swets, J. A. (1961). Is there a sensory threshold? Science, 134, 168 - 177.

Swets, J. A. (1979). ROC analysis applies to the evaluation of medical imaging techniques. Investigative Radiology, 14, 109 - 121.

Swets, J. A., Tanner, W. P., \& Birdsall, T. G. (1961). Decision processes in perception. Psychological Review, $68,301-340$. 